hep-ph/0506134

\title{
Prediction for the transverse momentum distribution of Drell-Yan dileptons at PANDA
}

\author{
O. Linnyk * K. Gallmeister, S. Leupold, and U. Mosel \\ Institut für Theoretische Physik, Universität Giessen, Germany
}

(Dated: October 26, 2018)

\begin{abstract}
We predict the triple differential cross section of the Drell-Yan process $p \bar{p} \rightarrow l^{+} l^{-} X$ in the kinematical regimes relevant for the upcoming PANDA experiment, using a model that accounts for quark virtuality as well as primordial transverse momentum. We find a cross section magnitude of up to $10 \mathrm{nb}$ in the low mass region. A measurement with $10 \%$ accuracy is desirable in order to constrain the partonic transverse momentum dispersion and the spectral function width within $\pm 50 \mathrm{MeV}$ and to study their evolution with $M$ and $\sqrt{s}$.

PACS numbers: 13.85.Qk, 12.38.Cy, 12.38.Qk
\end{abstract}

Using the model described and tested in an earlier publication [1], we calculate the $p_{T}$-spectrum of the DrellYan pairs in $\bar{p} p$ collision at the centre of mass energy $\sqrt{s}=5.5 \mathrm{GeV}$, which is an interesting, not yet experimentally explored kinematical regime. The planned experiment PANDA 2] at future GSI facility is to probe the Drell-Yan process at this energy.

The conventional perturbative QCD (pQCD) approach to the calculation of hard scattering cross sections assumes that the partons are collinear and on-shell, i.e. both the virtuality and transverse momenta of the quarks and gluons inside hadrons are neglected and the partonic four-momentum is replaced with it's light cone projection $\left(k^{+}, 0, \overrightarrow{0}\right)$. This approximation to the kinematics does not effect the cross section of fully inclusive processes like DIS, but it leads to unrealistic results for the cross sections of more exclusive processes. Intrinsic transverse momentum of quarks has been proven to be important

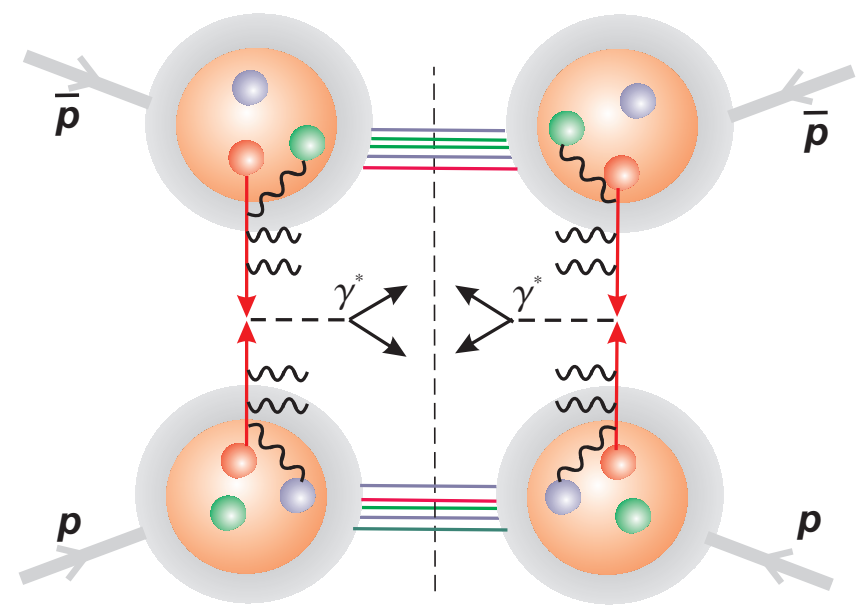

FIG. 1: Relevant initial state interactions that can build up intrinsic transverse momentum and width of quarks in the proton in the Drell-Yan process.

*Electronic address: olena.linnyk@theo.physik.uni-giessen.de for the interpretation of various cross sections and asymmetries [3]. The virtuality of quarks and gluons in the proton has attracted considerable attention recently. As shown in [1], by taking into account the virtuality distribution of partons one can explain the K-factor type corrections to the transverse momentum distribution of Drell-Yan pairs. The double differential cross section of the Drell-Yan process $d \sigma / d M d x_{F}$ can be well described by pQCD in the conventional collinear on-shell approach. However, the $p_{T}$ distribution, i.e. the triple differential cross section $d \sigma / d M d x_{F} d p_{T}$ in every order of collinear pQCD is unphysically sharp. One has either to resum all orders by the Sudakov method, introducing additional regularization parameters, or to correct the kinematics by taking into account quark transverse momentum and virtuality. The latter procedure leads to a very good description of data already in the leading order of pQCD for the elementary process [1]. The importance of accounting for the virtuality of partons in other high energy processes was stressed, for instance, by O. Benhar and V. R. Pandharipande [4] and, recently, by J. Collins and H. Jung [5].

The generalization of the usual parton distribution function, which depends on the virtuality and $k_{T}$ of the quark in addition to it's longitudinal momentum $k^{+}$, is sometimes called "double unintegrated parton density" or "parton correlation function". But the terminology is still not fixed, and one of the reasons is that the gauge invariant definition of these distributions in terms of parton field operators is not known yet. It is an open question, whether factorization still holds in the generalized case, in which the soft part of the cross section depends on the full quark momentum $\left(k^{+}, k^{-}, k_{T}\right)$. Also, the evolution of these parton distributions has not been studied so far. Before the factorization theorem is proven, one can calculate measurable cross sections using a model that assumes generalized factorization. Of course, the predictive power of this model has to be tested. Comparison of such calculations to the data in [1] allowed us to fix parameters of a simple factorized parametrization for the unintegrated quark density in the proton. In the present paper, we use the same parameters to calculate the cross section in the kinematics of the future 
PANDA experiment in order to obtain a first estimate for the counting rates and $p_{T}$-distributions expected at $\sqrt{s}=5.5 \mathrm{GeV}, M=1-4 \mathrm{GeV}$, which is on the edge of the non-perturbative regime. To this aim we also compare with the PYTHIA event generator [6].

In [1], a model to calculate the initial state interaction (ISI) effects in the Drell-Yan process was proposed. This model assumes, firstly, the generalized factorization and, secondly, that the soft part of the cross section can be approximated by a product of functions of the quark $k^{+}$ momentum, the transverse momentum, and the virtuality $m^{2} \equiv k^{+} k^{-}-\vec{k}_{T}^{2}$. We use a Gaussian $k_{T}$-distribution

$$
g\left(\vec{k}_{T}\right)=\frac{1}{4 \pi D^{2}} \exp \left(-\frac{\vec{k}_{T}^{2}}{4 D^{2}}\right)
$$

so that the mean squared partonic intrinsic transverse momentum $\left\langle\vec{k}_{T}^{2}\right\rangle=4 D^{2}$.

The authors of [4] pointed out the analogy between the more conventional collinear approach and the plane wave impulse approximation (PWIA) of many body theory. Corrections to the PWIA are due to initial and final state (FSI) interactions. ISI of active and spectator quarks generate quark virtuality and intrinsic transverse momentum (see Fig. 11). Calculations in the quarkdiquark model show that the partonic ISI corrections to the cross section of the unpolarized Drell-Yan process are not small [7]. However, one has to go beyond the single gluon exchange approximation of [7] in order to study the ISI effects quantitatively. At present, the quark virtuality distribution cannot be calculated from first principles QCD. Using analogy to many body theory, we parametrize the quark virtuality distribution as a Breit-Wigner curve of width $\Gamma$, which is extracted from data

$$
\mathrm{A}(m, \Gamma)=\frac{1}{\pi} \frac{\Gamma}{m^{2}+\frac{1}{4} \Gamma^{2}} .
$$

The exact off-shell kinematics and off-shell sub-process cross section (at leading order in $\alpha_{S}$ ) are used. The intrinsic- $k_{T}$ approach is a limiting case of our model at $\Gamma \rightarrow 0$.

The model was tested in 1 by comparing the triple differential cross section $d^{3} \sigma / d M^{2} d p_{T}^{2} d x_{F}$ of the processes $p p \rightarrow \mu^{+} \mu^{-}+X$ and $p d \rightarrow \mu^{+} \mu^{-}+X$ to the data of the experiments E866 [8] and E772 [9]. Here, $M$ denotes the invariant mass of the produced lepton pair, $x_{F}$ its Feynman variable and $p_{T}$ its transverse momentum. Both the shape and magnitude of the observed triple differential cross sections were well reproduced for reasonable values of $\Gamma \approx 200 \mathrm{MeV}$ without a need for a $K$-factor.

We apply the formalism derived in [1] to predict the cross section

$$
\frac{d \sigma}{d \vec{p}} \equiv \frac{2}{\pi \sqrt{s}} \frac{d \sigma}{d x_{F} d p_{T}^{2}}=\frac{2}{\pi \sqrt{s}} \int_{\text {bin }} \frac{d^{3} \sigma}{d x_{F} d p_{T}^{2} d M^{2}} d M^{2}
$$

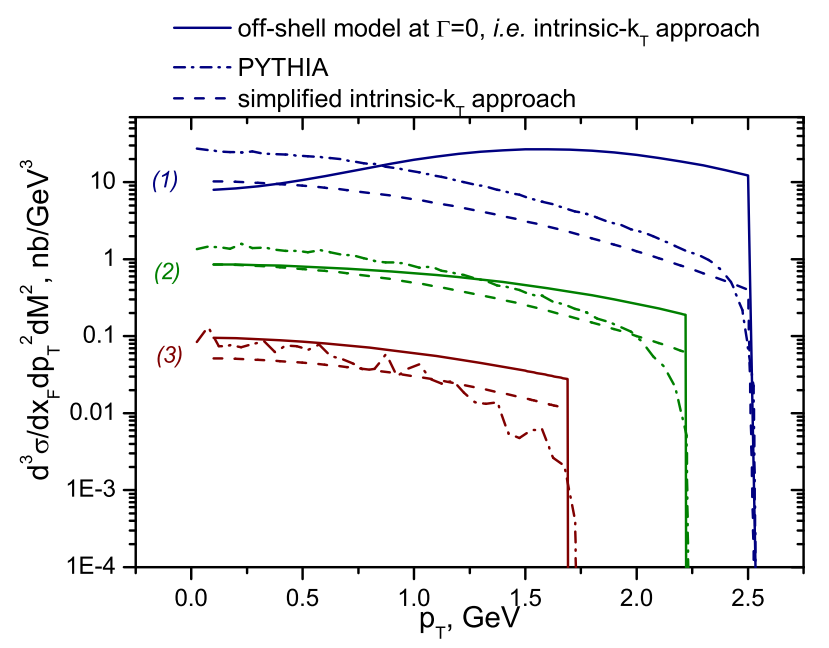

FIG. 2: Comparison of the predictions of different models: (solid) intrinsic- $k_{T}$ approach; (dash) intrinsic- $k_{T}$ approach with collinear sub-process cross section; (dash-dot) PYTHIA generator. $x_{F}=0, D=0.5 \mathrm{GeV}, \sqrt{s}=5.5 \mathrm{GeV}$. The lines marked with (1) correspond to $M=1 \mathrm{GeV}$; (2), $M=2 \mathrm{GeV}$; (3), $M=3 \mathrm{GeV}$.

of the process $\bar{p} p \rightarrow l^{+} l^{-} X$ in the kinematical regime planned to be probed by PANDA. We use

$$
\frac{d \sigma}{d x_{F} d p_{T}^{2} d M^{2}}=\sum_{\text {flavors }}\left(f_{p} \bar{f}_{\bar{p}}+f_{\bar{p}} \bar{f}_{p}\right) \otimes \frac{d \hat{\sigma}}{d x_{F} d p_{T}^{2} d M^{2}} \otimes \mathrm{A} \overline{\mathrm{A}}
$$

where $\sqrt{s}$ is the centre of mass energy of the $\bar{p} p$, the parton level cross section $d \hat{\sigma}$ is taken from [1], the spectral function $\mathrm{A}$ is given in (2), the unintegrated parton distribution $f$ is a product of (1) and the parton distribution functions [11], the quantities referring to anti-quarks are denoted with bars. We assume that $\bar{\Gamma}=\Gamma$ and $\bar{D}=D$.

The two parameters of the model $(D$ and $\Gamma)$ should be extrapolated to the values of $M=(1-4) \mathrm{GeV}$ and $\sqrt{s}=5.5 \mathrm{GeV}$ relevant for PANDA. In [1], we have performed fits to the Fermilab experiments E866 [8] and E772 [9] in different bins of $M$. From that, we estimate the parameter $D \approx 0.6 \pm 0.18 \mathrm{GeV}$. The large uncertainty of the extrapolation is reflected in our estimate of the parameter error. The model parameter $D$ should be understood as representing the summed effect of the transverse motion of partons inside the nucleon and of the perturbative corrections [12]: $D^{2}=D_{\text {intr }}^{2}+D_{\text {pert }}^{2}$. The transverse momentum coming from higher orders of collinear perturbation theory shows at constant $\tau \equiv M^{2} / s$ a linear dependence on $s$ in addition to the logarithmic dependence on $M^{2}$ [13]: $\left\langle p_{T}^{2}\right\rangle_{\text {pert }}=s \alpha_{S}\left(M^{2}\right) f(\tau)$. On the other hand, the non-perturbative $D_{\text {intr }}$ does not show a strong dependence on $s$. An analysis using the event generator PYTHIA 6] has shown that the perturbative corrections constitute only $0.1 \%$ of the $p_{T}$-spectrum of DrellYan pairs with $p_{T} \neq 0$ at PANDA kinematics. Thus, we set $D \approx D_{\text {intr }}$.

Fig. 2 presents a comparison of the predictions obtained with three different methods: 1) our model at 


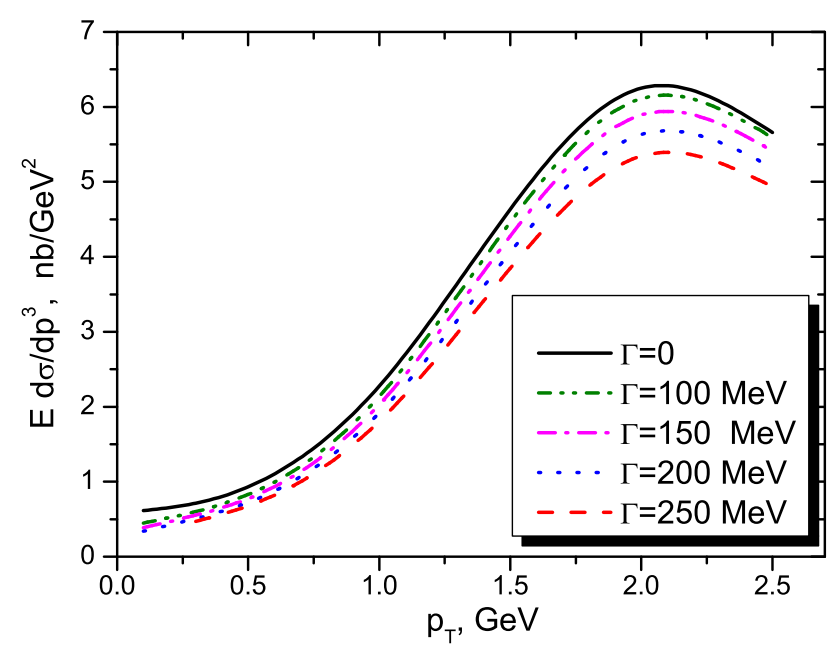

(a) $M=1 \mathrm{GeV}$

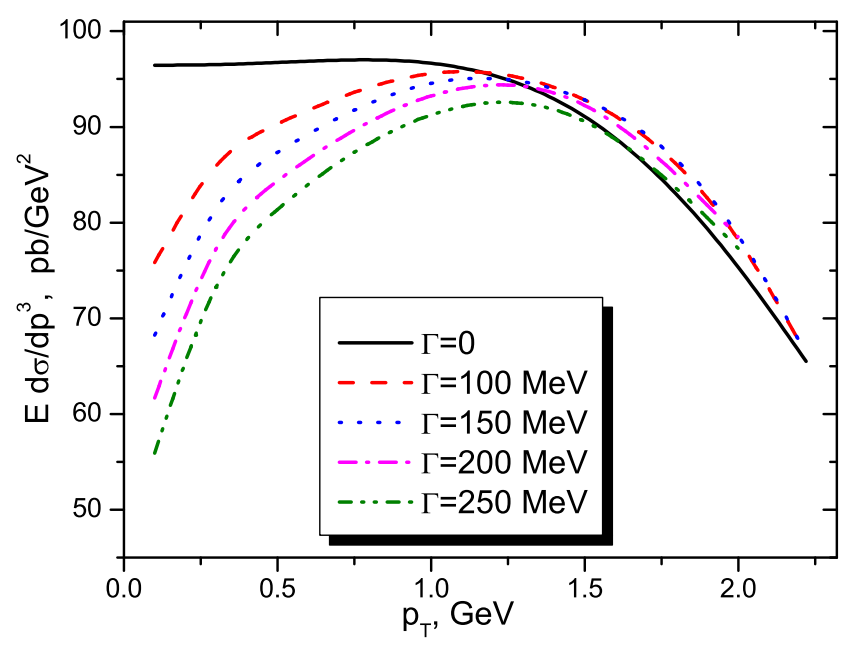

(b) $M=2 \mathrm{GeV}$

FIG. 3: Prediction for the $p_{T}$-distribution of Drell-Yan lepton pairs at PANDA. Intrinsic transverse momentum dispersion $D=0.6 \mathrm{GeV}$. The solid line is the result of calculations in the intrinsic- $k_{T}$ approach (width $\Gamma=0$ ). The other curves are generated with $\Gamma$ in the range that we have determined by fitting existing Drell-Yan data. Note that the scale changes from $\mathrm{nb}$ in the left figure to $\mathrm{pb}$ in the right one. $\sqrt{s}=5.5 \mathrm{GeV}, x_{F}=0.1$ in all plots.

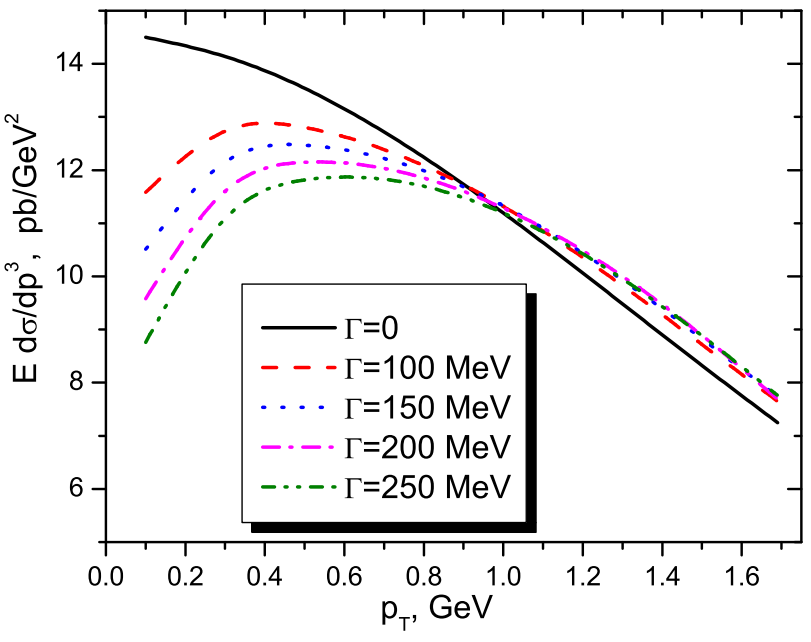

(a) $M=3 \mathrm{GeV}$

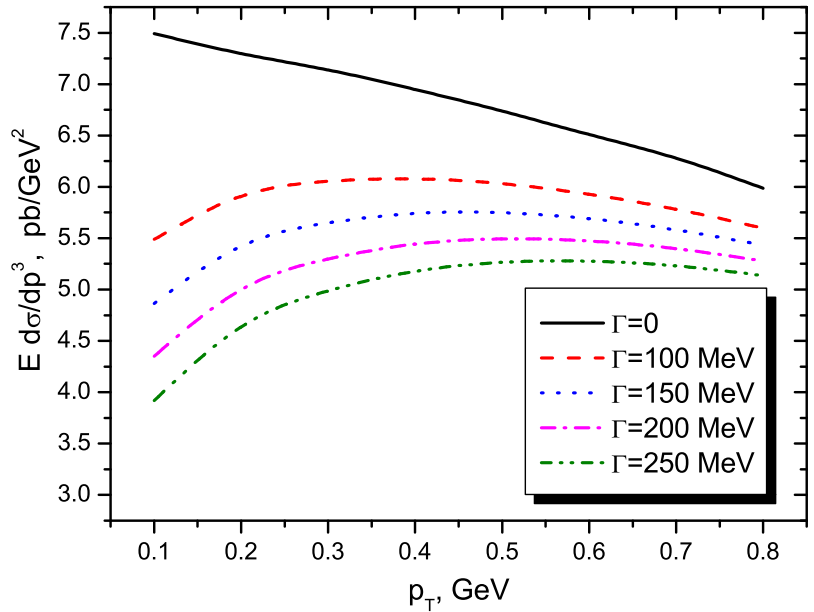

(b) $M=4 \mathrm{GeV}$

FIG. 4: Same as Fig. 3 but for higher masses of Drell-Yan pairs.

$\Gamma=0, D=0.5 \mathrm{GeV}$, which is equivalent to the intrinsic$k_{T}$ approach (solid lines); 2) the widely used simplified version of the intrinsic- $k_{T}$ approach, in which the dependence of $\hat{\sigma}$ on $k_{T}$ is neglected (dash line); 3) PYTHIA, taking into account QED and QCD initial state radiation and intrinsic- $k_{T}$ (dash-dotted line). The models agree in the overall strength of the cross section within a factor of 3. The demand of positive mass of the remnant $M_{X}$ determines the maximum $p_{T}$. In PYTHIA, however, a stronger constraint is implemented: $M_{X} \geq$ $2 m_{q q} \approx 1.6 \mathrm{GeV}$, where $m_{q q}$ is a diquark mass. We also used $1.6 \mathrm{GeV}$ as the lower bound for the remnant mass in the other models for consistency. The dilepton mass bin width in the simulation was set to $100 \mathrm{MeV}$. From Fig. 2] one observes that the calculations within the simplified intrinsic approach (dashed line) and within PYTHIA (dash-dotted line) give similar results. Indeed, the intrinsic- $k_{T}$ approach with an approximate collinear sub-process cross section is implemented in PYTHIA. The difference between the two curves (dash and dashdot) at high $p_{T}$ values are due to a more detailed modelling of the remnant in the event generator. Note the 


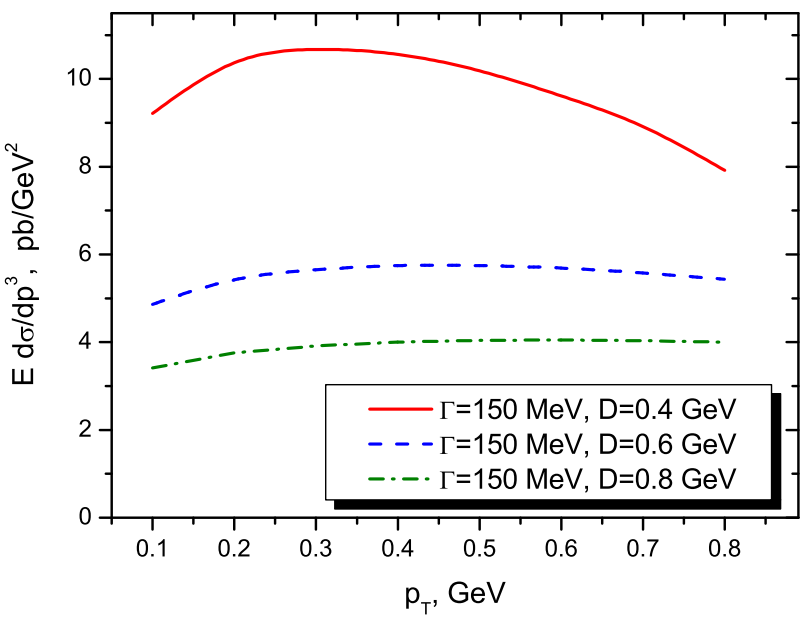

FIG. 5: Variation of the cross section with $D$ at fixed $\Gamma$. $s=32 \mathrm{GeV}^{2}, x_{F}=0.1, M=4 \mathrm{GeV}$.

qualitative difference of the cross section at the Drell-Yan pair mass $M=1 \mathrm{GeV}$ in the intrinsic- $k_{T}$ approach (solid lines). In contrast to the higher mass bins, the peak of the $p_{T}$-distribution for $M=1 \mathrm{GeV}$ is not at zero in our model (solid line in Fig. 2). This behavior appears at $M \lesssim 2 D$, i.e. in the distribution of the low virtuality photons, which can be produced by the partonic transverse motion alone. It is worthwhile to stress that this drastic change in the $p_{T}$-dependence of the cross section takes place for all values of $\Gamma$. An experimental verification of this effect would constitute a direct test for the transverse momentum distribution of partons. Note, however, that this effect appears only if the $k_{T}$-dependence of the partonic cross section is not neglected (this is the difference between the solid and dashed lines in Fig 2).

Fig. 3] and 4 show several theoretical curves for the cross section (3) with $\Gamma=(100-250) \mathrm{MeV}$, which is the range determined from fitting E866 data in 1]. The results of our calculations in the intrinsic- $k_{T}$ approach $(\Gamma=0)$ are plotted for comparison (solid lines). The evolution of the spectral function with the hard scale is unknown. It cannot be directly related to the evolution of the $k_{T}$-distribution, because the quark off-shellness depends also on $k^{-}$. One can see that the effect of the $\Gamma$ variation on the cross section is of the order of $10 \%$. On the other hand, the variation of the parameter $D$ within theoretical uncertainty at fixed $\Gamma$ (as presented in Fig. 5) also leads to considerable changes of the cross section. As was shown in 1], though, one can do a double fit and extract both parameters from the same data set.

The measurement of this cross section at $\sqrt{s}$ as low as $5.5 \mathrm{GeV}$ will provide essential information on QCD in the regime where effects beyond leading order and leading twist are expected to be large [14]. The high amplitude of the predicted cross sections (up to $10 \mathrm{nb}$ ) indicates that PANDA at the design luminosity has a potential to measure the triple differential unpolarized cross section of $\bar{p} p \rightarrow l^{+} l^{-}+X$ with high statistics and an unprecedented accuracy.

The transverse momentum spectrum of Drell-Yan pairs at low $\sqrt{s}$ is generated predominantly by the nonperturbative primordial intrinsic transverse momentum of the partons. On the other hand, the distribution of the intrinsic $k_{T}$ in this region is poorly known. PANDA data will be a valuable input that should allow one to pin down the quark transverse momentum distribution in the proton. Again, the $M=1 \mathrm{GeV}$ mass bin shows the mentioned shift of the peak towards higher $p_{T}$.

The presented results also suggest that one can use the future PANDA data to gain information on the spectral function of partons bound in the nucleon. Indeed, as we see from Fig. 3] and 4 an experimental accuracy of $20-30 \%$ would be enough to answer the question of whether the cross section can be described by a model with on-shell quarks. The results of the earlier calculations at higher $\sqrt{s}$ together with the PANDA data can be used also to extract the dependence of the quark spectral function in the proton on the hard scales $M$ and $\sqrt{s}$. For this purpose, an experimental accuracy of at least $10 \%$ is needed, so that one can reliably extract the parameter $\Gamma$ in different mass bins. Should the accuracy be even better, the data can be used to investigate the details of the quark spectral function shape.

Work supported by BMBF.
[1] O. Linnyk, S. Leupold and U. Mosel, Phys. Rev. D 71, 034009 (2005), arXiv:hep-ph/0412138.

[2] M. Kotulla et al., Technical Progress Report for PANDA, (2005), www.ep1.rub.de/ ${ }^{\sim}$ panda/archive/public/panda_tpr.pdf

[3] M. Fontannaz and D. Schiff, Nucl. Phys. B 132, 457 (1978); C.-Y. Wong and H. Wang, Phys. Rev. C 58, 376 (1998), arXiv:nucl-th/9802378; X.-N. Wang, Phys. Rev. C 61, 064910 (2000), arXiv:nucl-th/9812021; Y. Zhang, G. Fai, G. Papp, G. Barnafoldi, and P. Levai, Phys. Rev. C 65, 034903 (2002), arXiv:hep-ph/0109233; U. D'Alesio and F. Murgia, Phys. Rev. D 70, 074009 (2004) arXiv:hep-ph/0408092.
[4] O. Benhar, preprint, arXiv:nucl-th/0204042; M. W. Paris and V. R. Pandharipande, Phys. Lett. B 514, 361 (2001), arXiv:nucl-th/0105076.

[5] J. Collins and H. Jung, arXiv:hep-ph/0508280.

[6] T. Sjöstrand et al., Comput. Phys. Commun. 135, 238 (2001) arXiv:hep-ph/0010017.

[7] D. Boer, S. J. Brodsky, and D. S. Hwang, Phys. Rev. D 67, 054003 (2003), arXiv:hep-ph/0211110.

[8] J. C. Webb, arXiv:hep-ex/0301031.

[9] P. L. McGaughey et al., Phys. Rev. D 50, 3038 (1994), [Erratum-ibid. D 60119903 (1999)].

[10] E. Anassontzis et al., Phys. Rev. D 38, 1377 (1988).

[11] M. Glück, E. Reya, and A. Vogt, Eur. Phys. J. C 5, 461 
(1998), arXiv:hep-ph/9806404

[12] U. D'Alesio and F. Murgia, Phys. Rev. D 70, 074009 (2004) arXiv:hep-ph/0408092.

[13] G. Altarelli, G. Parisi and R. Pretonzio, Phys. Lett. B 76, 351 (1978); H. Fritzsch and P. Minkowski, Phys. Lett. B
73, 80 (1978)

[14] R. Hamberg, W. L. van Neerven and T. Matsuura, Nucl. Phys. B 359, 343 (1991) [Erratum-ibid. B 644, 403 (2002)]. 